\title{
The Application of P300-Long-Latency Auditory-Evoked Potential in Parkinson Disease
}

\author{
Natalia Ferrazoli ${ }^{1,2}$ Caroline Donadon ${ }^{2,3}$ Adriano Rezende ${ }^{2}$ Aiotr H. Skarzynski ${ }^{4,5}$ \\ Milaine Dominici Sanfins $2,6[0$
}

1 Postgraduate Program in Health, Interdisciplinary Practice and Rehabilitation, Universidade Estadual de Campinas, Campinas, SP, Brazil

${ }^{2}$ Clinica Ouvire, Campinas, SP, Brazil

3 Postgraduate Program in Child and Adolescent Health, Universidade Estadual de Campinas, Campinas, SP, Brazil

${ }^{4}$ Department of Teleaudiology and Hearing Screening,World Hearing Center, Institute of Physiology and Pathology of Hearing, Warsaw/ Kajetany, Poland

${ }^{5}$ Department of Heart Failure and Cardiac Rehabilitation, Institute of Sensory Organs, Warsaw, Poland

${ }^{6}$ Centro de Eletrofisiologia e Neuroaudiologia Avançada, Sao Paulo, SP, Brazil
Address for correspondence Caroline Donadon, Rua Goncalves Cesar 146, Jardim Guanabara, Campinas, São Paulo, Brazil (e-mail: caroldonadon.fono@gmail.com; carol_donadon@hotmail.com).

Int Arch Otorhinolaryngol 2022;26(1):e158-e166.

\begin{abstract}
Keywords

- Parkinson disease

- attention

- P300

- event-related potential

- elderly

Introduction Parkinson disease (PD) is a degenerative and progressive neurological disorder characterized by resting tremor, stiffness, bradykinesia, and postural instability. Despite the motor symptoms, PD patients also consistently show cognitive impairment or executive dysfunction. The auditory event-related potential P300 has been described as the best indicator of mental function, being highly dependent on cognitive skills, including attention and discrimination.

Objective To review the literature on the application and findings of P300 as an indicator of PD.

Data Analysis The samples ranged from 7 to 166 individuals. Young adult and elderly male patients composed most study samples. The Mini-Mental State Examination test, the Unified Parkinson Disease Rating Scale, and the Hoehn and Yahr Scale were used to assess neurological and cognitive function. In terms of testing hearing function, few studies have focused on parameters other than the P300. The factors we focused on were how the P300 was modified by cognitive effects, its correlation with different PD scales, the effect of performing dual tasks, the effect of fatigue, and the influence of drug treatments.

Conclusion The use of the P300 appears to be an effective assessment tool in patients with PD. This event-related potential seems to correlate well with other neurocognitive tests that measure key features of the disease.
\end{abstract}

received

September 12, 2020

accepted

November 8, 2020

published online

March 29, 2021
DOI https://doi.org/

10.1055/s-0040-1722250 ISSN 1809-9777.

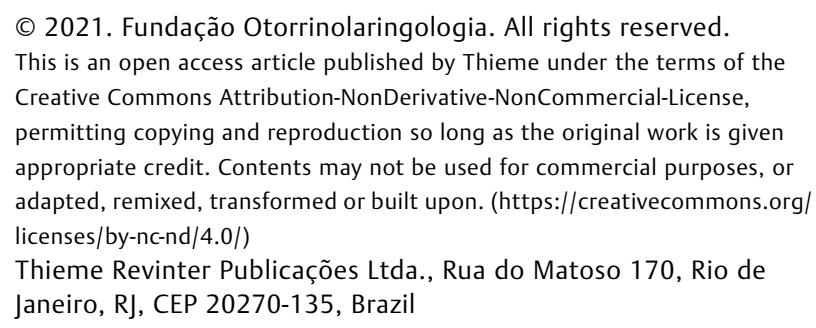
Creative Commons Attribution-NonDerivative-NonCommercial-License, permitting copying and reproduction so long as the original work is given appropriate credit. Contents may not be used for commercial purposes, or adapted, remixed, transformed or built upon. (https://creativecommons.org/ licenses/by-nc-nd/4.0/) Thieme Revinter Publicações Ltda., Rua do Matoso 170, Rio de Janeiro, RJ, CEP 20270-135, Brazil 


\section{Introduction}

Parkinson disease (PD) is a degenerative and progressive neurological disorder characterized by resting tremor, stiffness, bradykinesia, and postural instability. ${ }^{1}$ Despite being commonly associated with motor disorders, PD patients often show cognitive impairment or executive dysfunction, with difficulty starting tasks, lack of cognitive flexibility, dementia, lack of attention, and difficulty adapting to new stimuli. $^{2,3}$

The event-related potential (ERP) component known as the P300 is a neurophysiological parameter that has been found to correlate with cognitive processes, arising when an individual consciously recognizes a change in an auditory stimulus, a novel stimuli. ${ }^{4}$ The $\mathrm{P} 300$ potential identifies the positive component wave, with a peak around $300 \mathrm{~ms}$, which is generated after a novel sound stimulus.

By measuring an increase in latency, or sometimes a decrease in the amplitude of the P300 wave, it is possible to monitor the loss of cognitive functions associated with the processing of sound information. The measurement of such cognitive abilities as attention, discrimination, integration, memory, and decision-making can be performed. ${ }^{5}$

In view of the clinical use of the P300 potential to assess the cognitive skills in patients with degenerative and neurological disorders, the $\mathrm{P} 300$ has been proposed as a candidate biomarker for the progression of PD. The P300 is appreciably smaller in severe cases. ${ }^{6,7}$ The aim of the present study was to examine the use of P300 measurements in patients with PD and to identify consistent outcomes.
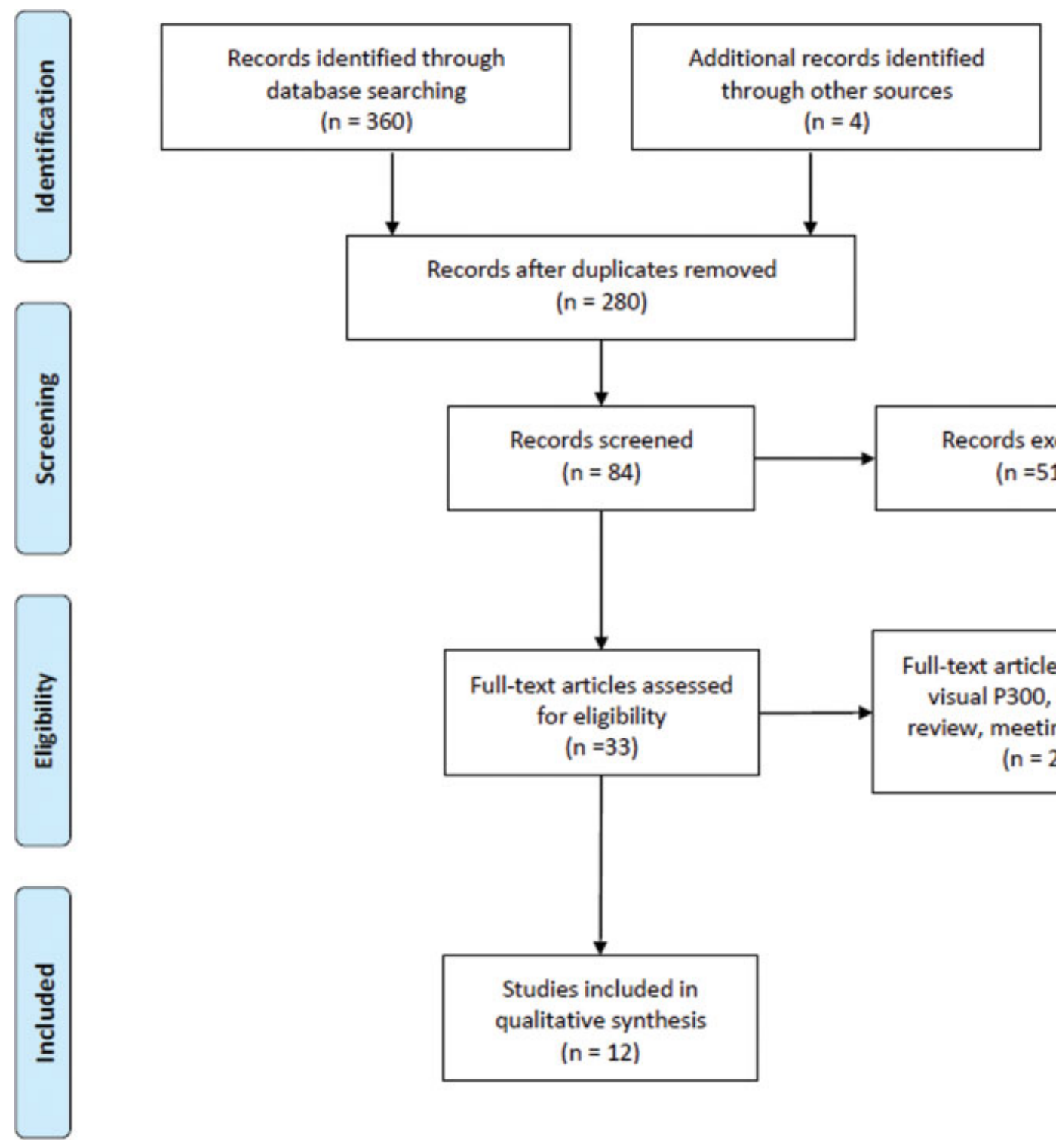

Records after duplicates removed

$(n=280)$
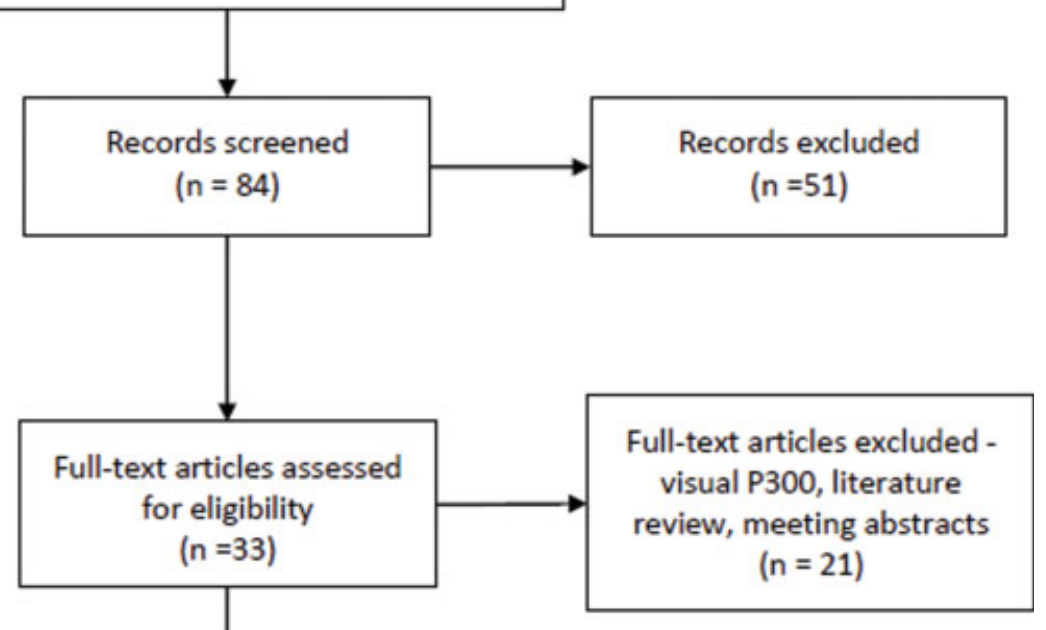

Fig. 1 Flowchart of the selection of the studies. 


\begin{tabular}{|c|c|c|c|c|c|}
\hline 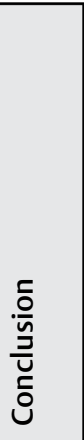 & 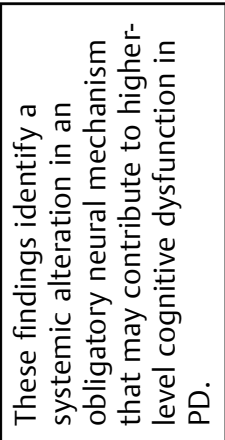 & 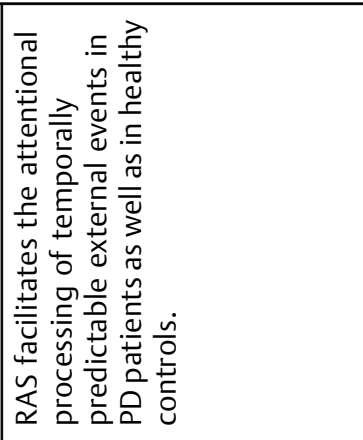 & 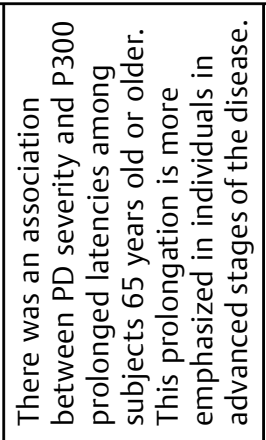 & 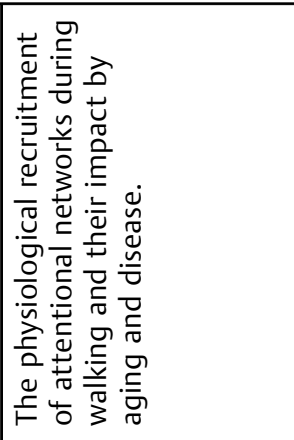 & 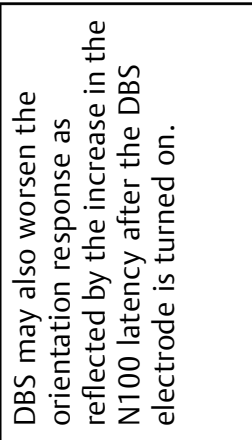 \\
\hline $\begin{array}{l}\frac{\tilde{y}}{\bar{y}} \\
\stackrel{y}{\simeq}\end{array}$ & 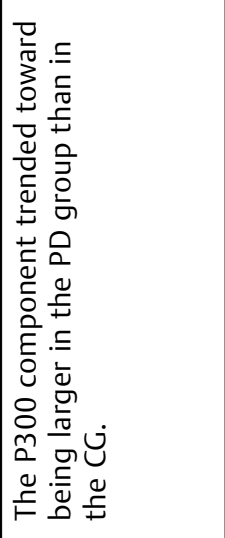 & 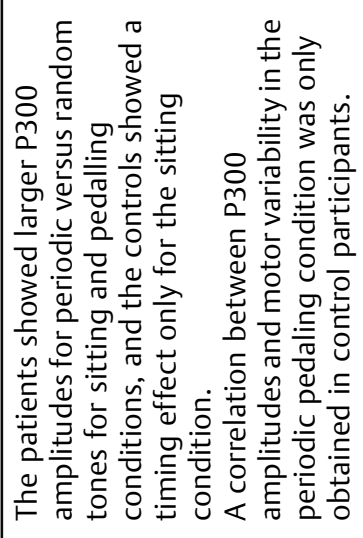 & 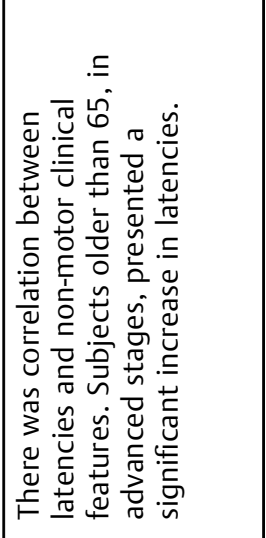 & 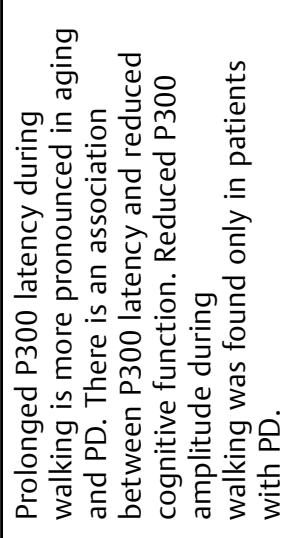 & 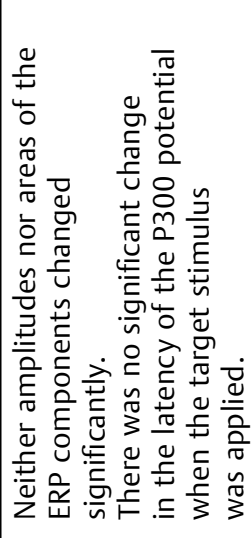 \\
\hline 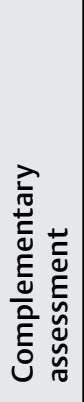 & 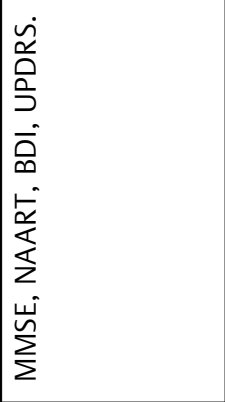 & 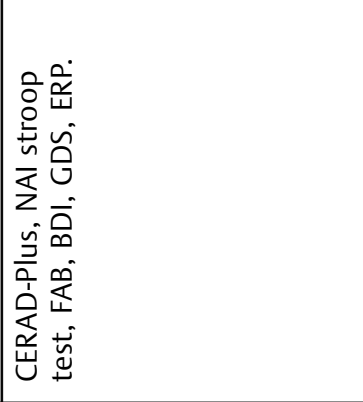 & 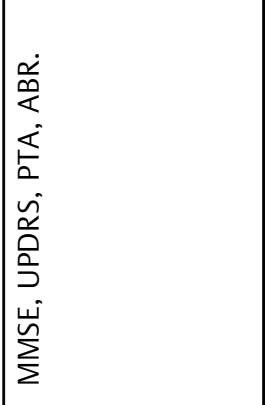 & 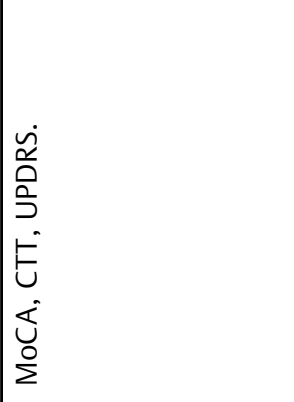 & 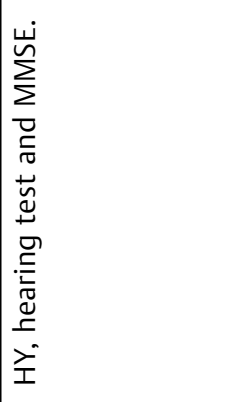 \\
\hline $\begin{array}{l}\frac{0}{0} \\
\frac{\hat{E}}{\tilde{N}} \\
\text { n }\end{array}$ & 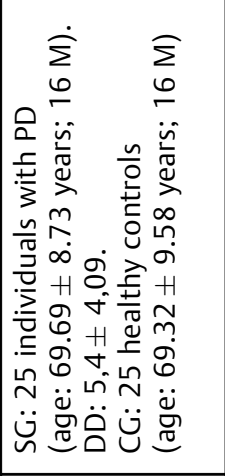 & 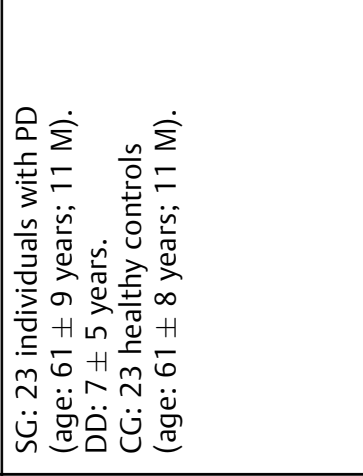 & 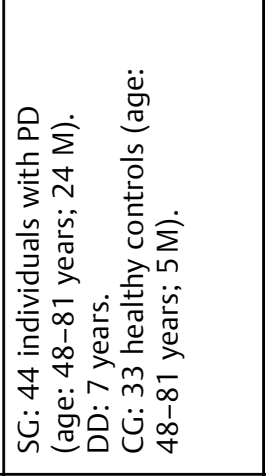 & 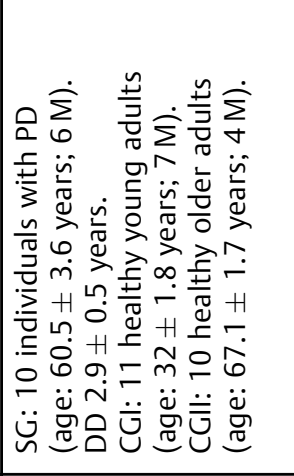 & 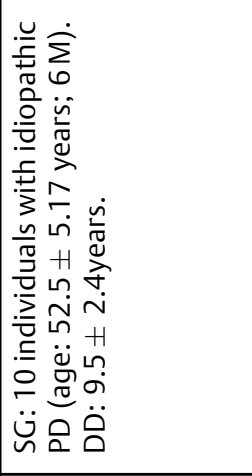 \\
\hline 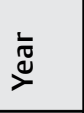 & $\stackrel{\infty}{\stackrel{\infty}{N}}$ & $\frac{0}{2}$ & $\underset{\sim}{\stackrel{\nabla}{v}}$ & $\frac{0}{0}$ & 웅 \\
\hline $\begin{array}{l}\frac{n}{0} \\
\frac{1}{5} \\
\frac{3}{<}\end{array}$ & 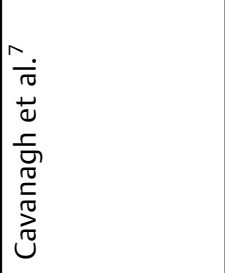 & $\mid \begin{array}{l}\frac{\infty}{\pi} \\
\frac{\pi}{\sigma} \\
-\bar{\omega} \\
\end{array}$ & 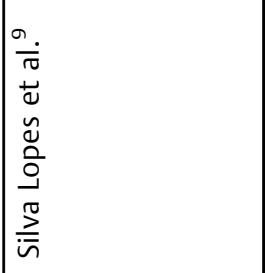 & 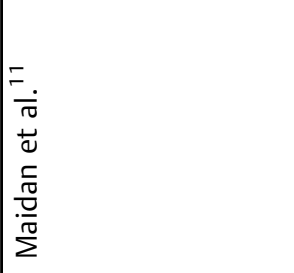 & 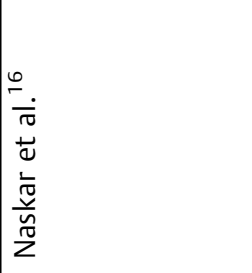 \\
\hline & - & $N$ & $m$ & $\nabla$ & in \\
\hline
\end{tabular}




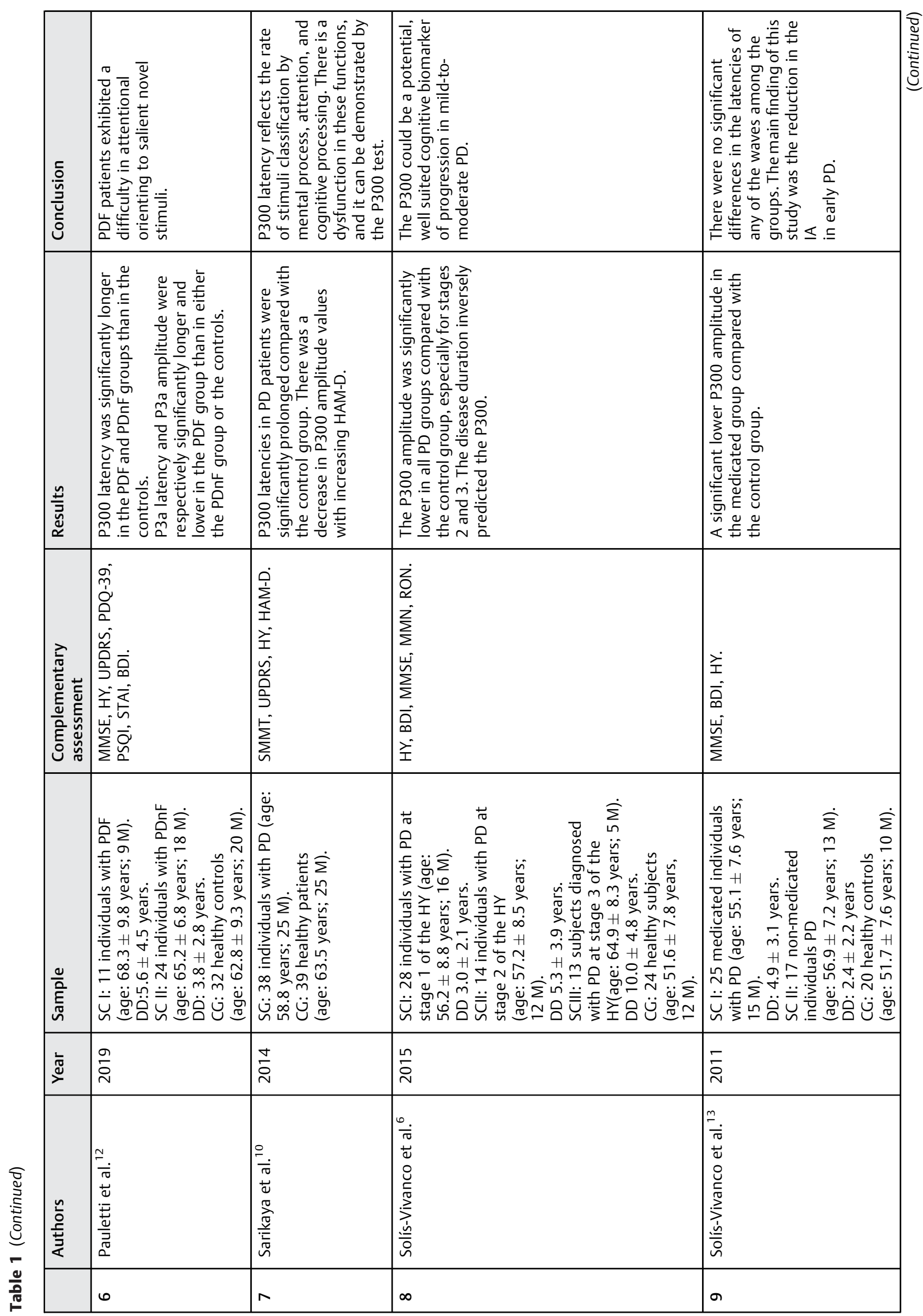



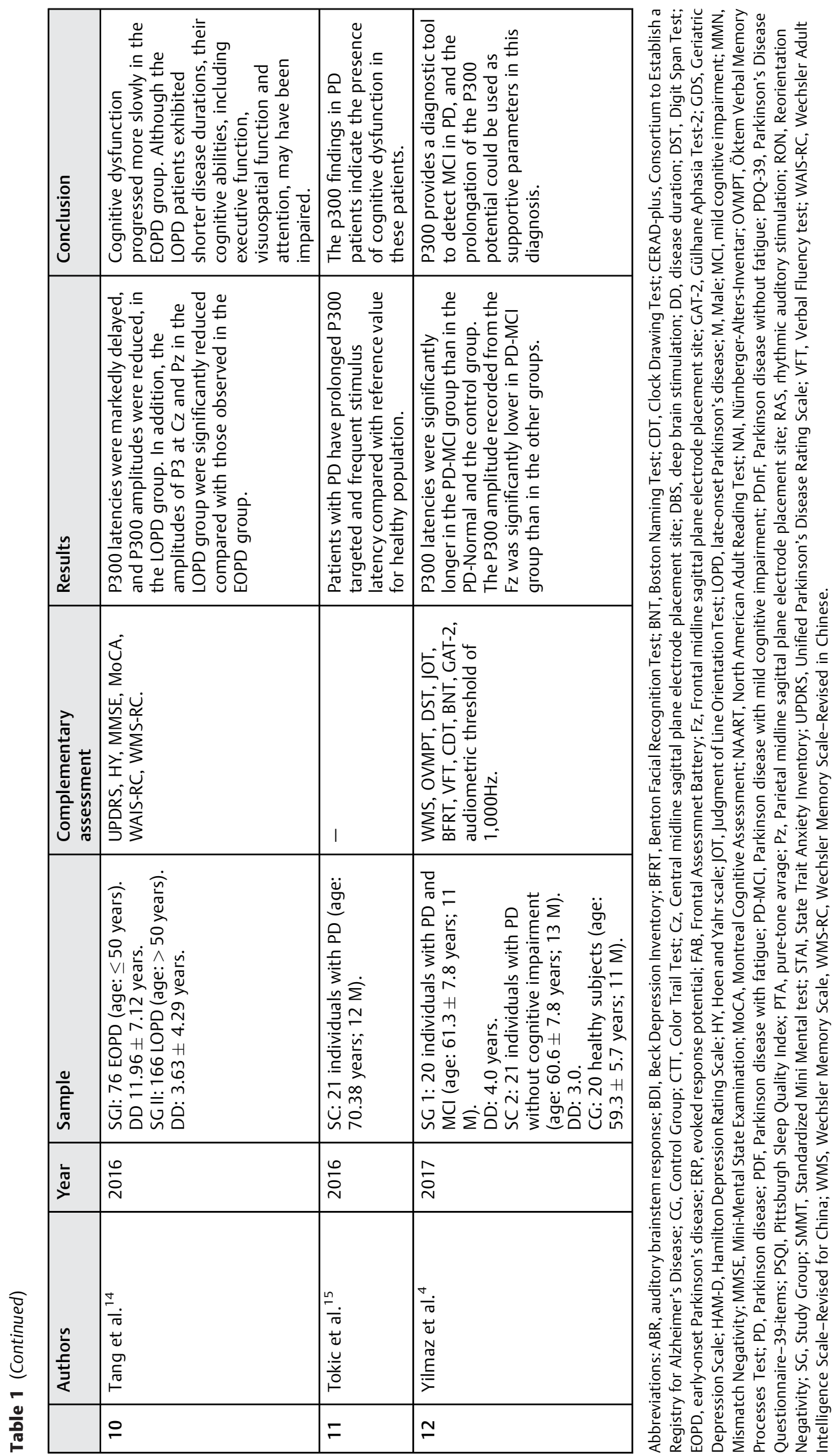


\section{Review of the Literature}

\section{Methodology}

A systematic review of the literature was performed in March 2020 on three databases (PubMed, Web of Science, and Scopus). We searched for full-length articles and abstracts using the Medical Subject Headings (MeSH) parkinson disease AND $p 300$ OR event related auditory potential OR long latency auditory evoked potential.

The titles and abstracts were evaluated by two independent researchers to check if they fitted the inclusion and exclusion criteria. The searches included studies published in the past 10 years (2010-20) which were original articles and tested for auditory P300 using pure-tone stimuli. Animal studies, meeting abstracts, and works in languages other than English were excluded. Disagreements were resolved by discussion and consensus among the authors. Information from the full-text remaining articles was inserted into a Microsoft Excel (Microsoft Corporation, Redmond, WA, US) spreadsheet.

\section{Result}

Out of the 360 initial studies (-Fig. 1), a total of 12 articles were included in the present review. Information about sample size and characteristics, complementary assessments, P300 findings, and the conclusions from all included articles are summarized in - Table $\mathbf{1}$.

\section{(i) Sample Characteristics}

In the 12 articles, most participants were adults or elderly (aged between 32 and 81 years) and male. Yilmaz et al. ${ }^{4}$ did not mention the age of their participants. The sizes of the samples ranged from 7 to 166 individuals, in both experimental and controls groups. Four articles used a control group composed of healthy age-matched patients, ${ }^{7-10}$ while five $\mathrm{e}^{4,6,11-13}$ used a control group composed of both healthy individuals and those with PD. The study by Tang et al. ${ }^{14}$ was composed only of individuals with PD, with different disease durations. Two studies ${ }^{15,16}$ did not include a control group in their methodology. A total of 9 of the articles (75\%) considered the time of manifestation and diagnosis of PD (disease duration), and the mean ranged from 2.9 to 9.5 years. ${ }^{6-9,11-14,16}$

\section{(ii) Neurological and cognitive assessments}

Another categorization was the neurological aspect of individuals with PD, factors including executive function, motor function, intelligence quotient (IQ), reasoning, language, memory, and praxis. The most used assessment tool in the studies was the Mini-Mental State Examination (MMSE), appearing in 7 articles, ${ }^{6,7,9,12-14,16}$ followed by the Unified Parkinson's Disease Rating Scale (UPDRS) ${ }^{7,9-12,14}$ and the Hoehn and Yahr (HY) staging scale,, $6,10,12-14,16$ in 6 articles.

In addition to the P300, other auditory assessments performed by the authors were the Auditory Brainstem Response (ABR), ${ }^{9}$ audiometric threshold at $1,000 \mathrm{~Hz},{ }^{4}$ auditory air thresholds at $0.5-8 \mathrm{kHz},{ }^{9}$ a hearing test, ${ }^{16}$ and selfreported normal auditory function. ${ }^{6}$ (iii) Cognitive ERP (P300) - latency and amplitude effects

The cognitive ERP reflects the time required for auditory processing, and it is frequently studied in patients suffering from PD. In one study, ${ }^{10}$ the P300 of subjects with PD had no systematic difference in amplitude; however, it did show a delay (increase in latency) when compared with healthy individuals. $9,10,15$

According to the findings of Yilmaz et al., ${ }^{4}$ cognitive impairment seemed to affect P300 latency, causing a prolongation in PD patients with mild cognitive impairment (MCI-PD) when compared with DP-Normal and control groups. In that same study, ${ }^{4}$ when the P300 latency of PD patients with no cognitive impairment was compared with latencies from healthy controls, no difference was found.

Other variables related to PD seem to be associated with changes in the P300 latency and amplitude. Age at the onset of the disease, ${ }^{14}$ disease duration, ${ }^{15}$ and disease stage ${ }^{9}$ seem to have an effect.

When P300 latencies in a group with early onset of PD (at or before the age of 40) were compared with the latencies of a late-onset group (at or after the age of 41), delayed latencies and reduced amplitudes were observed in the late-onset group. ${ }^{14}$ These results confirm the negative correlation found between age, P300 latency, and amplitude in PD patients in other studies. $^{10,15}$

Disease duration can also impact the P300, since the longer the duration of the disease, the lower the amplitude values, ${ }^{6}$ and the more delayed the latencies. ${ }^{15}$ In contrast, another article $^{10}$ found no significant differences in PD duration and P300 amplitude and latency.

\section{(iv) PD scales and P300}

In one study, ${ }^{6}$ the P300 had lower amplitudes in all PD groups compared with the control group, especially for stages 2 and 3 on the HY scale. As for P300 latency, there was one finding of a positive correlation with the severity of PD in individuals aged 65 and older. ${ }^{9}$ Notwithstanding, Sarikaya et al. $^{10}$ found no significant correlation between HY scale scores and P300 amplitude and latencies.

One study ${ }^{9}$ that used the UPDRS scale to measure PD progress found a positive correlation between P300 latency and stage I and II PD patients. Using the same scale, to measure motor and global scores, Sarikaya et al. ${ }^{10}$ found no significant difference in the values of P300 latency and amplitude.

\section{(v) Dual tasks and fatigue in P300}

Other authors have focused on understanding how the auditory-motor pathway behaves in PD by measuring the P300 during the performance of dual motor tasks 8,11 and during fatigue conditions. ${ }^{12}$ In investigating P300 changes during an odd-ball auditory task between two conditions (that is, standing and walking), the results showed prolonged P300 latency during walking compared with standing in all groups, while P300 amplitude was similar between the two conditions. ${ }^{11}$ During the walking task, P300 latency was significantly shorter in the healthy young subjects compared with the healthy older adults and the patients with PD, but no 
differences were observed between the healthy older adults and the patients with PD. In the standing task, differences in P300 latency were only observed between healthy young subjects and patients with PD. ${ }^{11}$ As for P300 amplitude, smaller values were found while walking compared with standing in patients with PD. ${ }^{11}$

In the cognitive domain, PD patients had larger P300 amplitudes for rhythmic auditory stimulation (RAS) versus random tones for sitting and pedalling conditions. The controls showed an amplitude effect only for the sitting condition, but not for the pedalling condition. However, a correlation between P300 amplitudes and motor variability in the periodic pedalling condition was only obtained in control participants. ${ }^{8}$

Although recent studies ${ }^{17-19}$ have improved our understanding of fatigue, data on the pathophysiological mechanisms underlying this symptom are not yet unequivocal. Central fatigue may be associated with cognitive deficits in PD. ${ }^{12}$ P300 latency was significantly longer, and amplitude was lower, in patients with PD and fatigue than in either those with PD but no fatigue, or the controls. ${ }^{12}$

\section{(vi) PD treatments and P300}

The P300 has also been used to examine the cognitive effects on the brain in individuals with PD to understand the effects of drug treatments. ${ }^{7,13,16}$ Solís-Vivanco et al. ${ }^{13}$ observed a difference in P300 amplitude in PD patients who were being treated with at least two anti-parkinsonian drugs. There was a lower amplitude in the drug group compared with the healthy control group. When the group with non-medicated PD was compared with the control group, the amplitudes were lower but not statistically significant.

In contrast, another study ${ }^{7}$ found statistical differences between the PD and control groups, with greater P300 amplitudes in the PD group (with and without medication) compared with the control group.

In addition to drugs, deep brain stimulation (DBS) surgery and its possible changes in the ERP have been investigated. Naskar et al. ${ }^{16}$ investigated recordings of patients with the DBS on and off, and found no statistically significant differences in P300 latencies or amplitudes. However, for the DBSon condition, P300 latencies became slightly shorter, and the amplitudes also showed a slight decline; however these changes were not statistically significant.

\section{Discussion}

Parkinson disease is among the most common neurodegenerative diseases, preceded only by Alzheimer disease. Its signs and symptoms progress over time, so age is clearly a large factor in PD expression. The prevalence of PD is observed mostly in male individuals, and is positively correlated with age, being more prevalent in adults older than 60 years of age, although it can affect adults under the age of 40 , corroborating the data collected in the present review. ${ }^{14}$

The diagnosis of PD does not yet have a widely accepted objective measure, which is why it is largely clinical, ${ }^{20}$ commonly made by observing indications in the motor and non-motor fields. Diagnostic accuracy can be improved with the use of standardized clinical criteria such as the UPDRS $^{7,9-12,14}$ and the HY scale. ${ }^{6,10,12-14,16}$

The scores found on the UPDRS and HY scales correlated positively with the latency values. ${ }^{9,13}$ The UPDRS and HY scores assess losses in mental activity, behavior, mood, and signs and symptoms, factors that allow the individual to be classified in terms of their level of disability, which, in turn, have an effect on generation of the P300. ${ }^{21}$

Researchers presume that the $\mathrm{P} 300$ potential might be an important neurophysiological factor associated with cognitive functions such as decision-making, attention, discrimination, integration, and memory ${ }^{5}$-skills that are commonly altered in PD patients. ${ }^{2,3}$ This assumption leads us to suppose that the correlations found between scales and latency values might be explained by underlying cognitive, attentional, and executive functions which are involved in the planning and execution of daily-life activities.

However, there are some studies ${ }^{10,22}$ that do not fit the pattern. They fail to verify a relationship between the scales to assess PD and the values of latency and amplitude. Perhaps these anomalies can be explained by the existence of different dopaminergic pathways related to motor and mental impairment in $\mathrm{PD},{ }^{9}$ and by the fact that the same score on one scale can originate from different clinical manifestations depending on the individual.

Cognitive decline (dementia) is one of the main changes in upper cortical function that can manifest in $30 \%$ to $40 \%$ of PD patients. Some degree of cognitive impairment is present at any stage of the disease; for this reason, early cognitive investigation is recommended, ${ }^{23,24}$ as observed in the studies $^{6,7,9,12-14,16}$ that used MMSE to assess cognitive factors.

As observed by Yilmaz et al., ${ }^{4}$ the absence of a statistical difference between P300 latency in a group with PD without cognitive impairment and another group of healthy control individuals can be explained by the hypothesis that frontal executive losses, clearly evident at the beginning of PD, may be more clearly related to altered prefrontal dopaminergic activity, and not necessarily to dementia. ${ }^{25}$

The P300 latency reflects the speed of auditory processing of external stimuli, cognition, and memory capacity, whereas amplitude values define the quality of the auditory information process, as they relate to the number of neurons and attentional resources activated during the task. ${ }^{4,11,26}$ There is a general consensus that $\mathrm{P} 300$ latency increases with age due to the aging of nervous system structures, even in non-pathological individuals; it also increases with a reduction in the cognitive ability to allocate attention and memory resources. $9,10,14$

The relationship between the clinical stage of the disease, the duration of the disease, and the age of the patient at its onset can be elucidated by the dopaminergic influence, and the detection of new/rare stimuli has been related to the fronto-striatal functioning ${ }^{27}$ suffered by P300. These criteria are related to PD disorders, since, during the initial stage of $\mathrm{PD}$, neurodegeneration is less severe and less extensive than at the most severe moment of the disease, ${ }^{28}$ which supports the findings of delayed latency and decreased P300 amplitude observed in PD patients. 
Regarding P300 amplitude, some studies ${ }^{6,14}$ indicate a decrease in amplitude with increasing age, while others report no clear evidence of the effect of age or disease duration. ${ }^{10}$ This variation in findings can be explained by the wide variability in the values found in the analyses. Considering these findings, latency values seem to be more sensitive to small cognitive fluctuations in PD and likely to be influenced by changes in the dopaminergic levels in the brain.

Some theories suggest that motor activities (such as walking or cycling) require higher cognitive processes that use a complex neural network that incorporates cognitive and motor information. ${ }^{29,30}$ Studies ${ }^{11,30}$ show that the addition of a simultaneous task of executive attention function to the task of walking (motor act) leads to changes in gait performance, and that this effect is exacerbated with aging and neurodegenerative diseases, such as PD.

The prolongation of P300 latency in walking condition in elderly individuals and in those with $\mathrm{PD}^{11}$ suggests an effect of aging or neurodegeneration, causing the lower processing speed to be accentuated in a more complex task, such as walking. The smaller amplitude only observed in the group with PD when comparing the walking and standing conditions raises the hypothesis that the amount of resources involved during the execution of the double task in PD individuals indicates a lower recruitment of attentional resources and a lower activation of neurons during information processing. ${ }^{31}$

Rhythmic auditory stimulation is defined as a therapeutic application of pulsed rhythmic or musical stimulation to improve gait or aspects related to movement. ${ }^{32}$ It has been shown that it can assist in the treatment of issues related to the motor domain, ${ }^{33,34}$ improving the spatio-temporal characteristics of gait, and cognitive processes, through its temporal predictability ${ }^{33}$ in patients with PD.

The findings of Lei et al. $^{8}$ indicate that RAS facilitates the processing of predictable events in patients with PD, as well as in healthy individuals, being observed by the increase in the amplitude of responses. The predictability of presenting RAS may have facilitated attentional processing in individuals with PD, since attention can be directed with greater precision at instants close to the appearance of the stimulus. $^{8,33}$ However, no correlation was found between P300 amplitudes and motor variability (sitting still or pedalling) in individuals with PD, only in the control group. The accuracy of the sensorimotor synchronization task may be associated with the functioning of the basal ganglia, ${ }^{35}$ the worsening of age-related motor functioning, and a negative effect of added cognitive tasks. ${ }^{36}$

Fatigue is a motor symptom that affects more than $50 \%$ of the parkinsonian population, ${ }^{37}$ consisting predominantly of central fatigue, ${ }^{38}$ which has been linked by researchers to cognitive $^{19,37}$ and attention ${ }^{12,18}$ deficits in PD. Longer latency and a decrease in amplitude in PD patients compared with controls suggests that they take longer to assess and detect the target stimulus. ${ }^{12}$

However, when comparing parkinsonian patients with fatigue and without fatigue, their latencies were equally prolonged, without statistical significance, which suggests that the fatigue mechanisms and the top-down mechanisms of attentional discrimination of the stimulus are not correlated. ${ }^{12}$ This provides indirect evidence of the importance of the connection between cognitive and motor functions during walking. ${ }^{11}$ There is an increase in the activation of the prefrontal cortex in young people and healthy elderly people during dual-task walking compared with normal walking. ${ }^{39-41}$ Patients with PD showed similar findings, but also showed greater activation during normal walking, ${ }^{42}$ suggesting a dependence on cognitive resources even in simple tasks. ${ }^{11}$

For the treatment of PD, dopaminergic agents are generally used to restore the missing neurotransmitter and improve clinical deficits. ${ }^{43}$ However, the relationship between the P300, the antiparkinsonian treatment, and PD is still nonspecific. Data indicate either an increase in amplitude $^{7}$ or a decrease ${ }^{13}$ when individuals undergoing drug treatment are compared with a control group. Authors 7,13 have hypothesized that, in addition to the use of drugs, the stage of the disease may also be a factor to consider. Therefore, the detection of the target stimulus may result in a larger P300 when the disease is more advanced. ${ }^{13}$

Several drugs can treat the symptoms of PD, but, in the long term, the patients will experience the motor complications induced by levedopa. ${ }^{16,44}$ Deep brain stimulation has been reported as a procedure that can improve both motor symptoms and cognitive aspects. ${ }^{16,45}$ However, the P300 elicited during DBS-on and DBS-off conditions did not show statistically significant changes in latency or amplitude. This finding correlates with the fact that the P300 potential is produced by a series of generators that are not affected by subthalamic nuclei projections. ${ }^{16}$

\section{Final Comments}

The use of the P300 can be very effective for patients with PD, since this ERP seems to be a good candidate for neurocognitive research in PD. The P300 appears sensitive to the duration and severity of the disease, and it shows marked differences for dual tasks and among the various treatment options applied to PD patients.

\section{Conflict of Interests}

The authors have no conflict of interests to declare.

\section{References}

1 Macuglia GR, Rieder CRM, Almeida RMM. Executive Functions in Parkinson's Disease: Systematic Review of Literature. PSICO 2012; 43(04):552-561

2 Robbins TW, Cools R. Cognitive deficits in Parkinson's disease: a cognitive neuroscience perspective. Mov Disord 2014;29(05): 597-607

3 Dirnberger G, Jahanshahi M. Executive dysfunction in Parkinson's disease: a review. J Neuropsychol 2013;7(02):193-224

4 Yilmaz FT, Özkaynak SS, Barçin E. Contribution of auditory P300 test to the diagnosis of mild cognitive impairment in Parkinson's disease. Neurol Sci 2017;38(12):2103-2109

5 Kraus N, MCGee T. Auditory event related potentials. Handbook of Clinical Audiology Baltimore: Williams \& Wilkins; 1994 
6 Solís-Vivanco R, Rodríguez-Violante M, Rodríguez-Agudelo Y, Schilmann A, Rodríguez-Ortiz U, Ricardo-Garcell J. The P3a wave: A reliable neurophysiological measure of Parkinson's disease duration and severity. Clin Neurophysiol 2015;126(11): 2142-2149

7 Cavanagh JF, Kumar P, Mueller AA, Richardson SP, Mueen A. Diminished EEG habituation to novel events effectively classifies Parkinson's patients. Clin Neurophysiol 2018;129(02):409-418

8 Lei J, Conradi N, Abel C, et al. Cognitive effects of rhythmic auditory stimulation in Parkinson's disease: A P300 study. Brain Res 2019;1716:70-79

9 Silva Lopes Md, Souza Melo Ad, Nóbrega AC. Delayed latencies of auditory evoked potential P300 are associated with the severity of Parkinson's disease in older patients. Arq Neuropsiquiatr 2014;72 (04):296-300

10 Sarikaya S, Yoldas TK, Yavasoglu NG. Evaluation of cognitive functions in Parkinson's patients without dementia with auditory event related potential (P300). Dusunen Adam 2014;27(02): 132-137

11 Maidan I, Fahoum F, Shustak S, et al. Changes in event-related potentials during dual task walking in aging and Parkinson's disease. Clin Neurophysiol 2019;130(02):224-230

12 Pauletti C, Mannarelli D, Locuratolo N, Currà A, Marinelli L, Fattapposta F. Central fatigue and attentional processing in Parkinson's disease: An event-related potentials study. Clin Neurophysiol 2019;130(05):692-700

13 Solís-Vivanco R, Ricardo-Garcell J, Rodríguez-Camacho M, et al. Involuntary attention impairment in early Parkinson's disease: an event-related potential study. Neurosci Lett 2011;495(02):144-149

14 Tang H, Huang J, Nie K, et al. Cognitive profile of Parkinson's disease patients: a comparative study between early-onset and late-onset Parkinson's disease. Int J Neurosci 2016;126(03):227-234

15 Tokic K, Titlic M, Beganovic-Petrovic A, Suljic E, Romac R, Silic S. P300 Wave Changes in Patients with Parkinson's Disease. Med Arh 2016;70(06):453-456

16 Naskar S, Sood SK, Goyal V. Effect of acute deep brain stimulation of the subthalamic nucleus on auditory event-related potentials in Parkinson's disease. Parkinsonism Relat Disord 2010;16(04): 256-260

17 Kenangil G, Orken DN, Ur E, Forta H, Celik M. The relation of testosterone levels with fatigue and apathy in Parkinson's disease. Clin Neurol Neurosurg 2009;111:412-414

18 Kluger BM, Pedersen KF, Tysnes OB, Ongre SO, Øygarden B, Herlofson $\mathrm{K}$. Is fatigue associated with cognitive dysfunction in early Parkinson's disease? Parkinsonism Relat Disord 2017;37:87-91

19 Goldman JG, Stebbins GT, Leung V, Tilley BC, Goetz CG. Relationships among cognitive impairment, sleep, and fatigue in Parkinson's disease using the MDS-UPDRS. Parkinsonism Relat Disord 2014;20:1135-1139

20 Homayoun H. Parkinson Disease. Ann Intern Med 2018;169(05): ITC33-ITC48

21 Mello MPB, Botelho ACG. Correlation of evaluation scales utilized at Parkinson's disease applied to physical therapy. Fisioter Mov 2010;23:121-127

22 Sohn YH, Kim GW, Huh K, Kim JS. Dopaminergic influences on the P300 abnormality in Parkinson's disease. J Neurol Sci 1998; 158:83-87

23 Aarsland D, Kurz MW. The epidemiology of dementia associated with Parkinson's disease. Brain Pathol 2010;20(03):633-639

24 Peavy GM. Mild cognitive deficits in Parkinson disease: where there is bradykinesia, there is bradyphrenia. Neurology 2010;75 (12):1038-1039

25 Mhyre TR, Boyd JT, Hamill RW, Maguire-Zeiss KA. Parkinson's disease. Subcell Biochem 2012;65:389-455
26 Katsarou Z, Bostantjopoulou S, Kimiskidis V, Rossopoulos E, Kazis A. Auditory event-related potentials in Parkinson's disease in relation to cognitive ability. Percept Mot Skills 2004;98(3 Pt 2):1441-1448

27 Boot N, Baas M, van Gaal S, Cools R, De Dreu CKW. Creative cognition and dopaminergic modulation of fronto-striatal networks: Integrative review and research agenda. Neurosci Biobehav Rev 2017;78:13-23

28 Kalia LV, Lang AE. Parkinson disease in 2015: Evolving basic, pathological and clinical concepts in PD. Nat Rev Neurol 2016;12 (02):65-66

29 Woollacott M, Shumway-Cook A. Attention and the control of posture and gait: a review of an emerging area of research. Gait Posture 2002;16(01):1-14

30 Yogev-Seligmann G, Hausdorff JM, Giladi N. The role of executive function and attention in gait. Mov Disord 2008;23(03):329-342, quiz 472

31 Johnson R Jr. On the neural generators of the P300 component of the event-related potential. Psychophysiology 1993;30(01): 90-97

32 Thaut C, Rice R. Rhythmic Auditory Stimulation (RAS). In: Thaut M, Hoemberg V, ed. Handbook of Neurologic Music Therapy. Oxford: Oxford University Press; 2014:94-105

33 Bella SD, Benoit CE, Farrugia N, Schwartze M, Kotz SA. Effects of musically cued gait training in Parkinson's disease: beyond a motor benefit. Ann N Y Acad Sci 2015;1337:77-85

34 Arias P, Cudeiro J. Effects of rhythmic sensory stimulation (auditory, visual) on gait in Parkinson's disease patients. Exp Brain Res 2008;186(04):589-601

35 van der Steen MC, Schwartze M, Kotz SA, Keller PE. Modeling effects of cerebellar and basal ganglia lesions on adaptation and anticipation during sensorimotor synchronization. Ann N Y Acad Sci 2015;1337:101-110

36 Nombela C, Hughes LE, Owen AM, Grahn JA. Into the groove: can rhythm influence Parkinson's disease? Neurosci Biobehav Rev 2013;37(10 Pt 2):2564-2570

37 Friedman JH, Brown RG, Comella C, et al;Working Group on Fatigue in Parkinson's Disease. Fatigue in Parkinson's disease: a review. Mov Disord 2007;22(03):297-308

38 Chaudhuri A, Behan PO. Fatigue and basal ganglia. J Neurol Sci 2000;179((S 1-2)):34-42

39 Holtzer R, Mahoney JR, Izzetoglu M, Wang C, England S, Verghese J. Online fronto-cortical control of simple and attention-demanding locomotion in humans. Neuroimage 2015;112:152-159

40 Metzger FG, Ehlis AC, Haeussinger FB, et al. Functional brain imaging of walking while talking - An fNIRS study. Neuroscience 2017;343:85-93

41 Mirelman A, Maidan I, Bernad-Elazari H, Shustack S, Giladi N, Hausdorff JM. Effects of aging on prefrontal brain activation during challenging walking conditions. Brain Cogn 2017;115:41-46

42 Maidan I, Nieuwhof F, Bernad-Elazari H, et al. The Role of the Frontal Lobe in Complex Walking Among Patients With Parkinson's Disease and Healthy Older Adults: An fNIRS Study. Neurorehabil Neural Repair 2016;30(10):963-971

43 Zhuang X, Mazzoni P, Kang UJ. The role of neuroplasticity in dopaminergic therapy for Parkinson disease. Nat Rev Neurol 2013;9(05):248-256

44 Deuschl G, Schade-Brittinger C, Krack P, et al;German Parkinson Study Group, Neurostimulation Section. A randomized trial of deep-brain stimulation for Parkinson's disease. N Engl J Med 2006;355(09):896-908

45 Barboza E Barbosa EN, Fichman HC. How is cognition in subthalamic nucleus deep brain stimulation Parkinson's disease patients? Dement Neuropsychol 2019;13(04):367-377 